\title{
Gender-Specific Left-Right Asymmetries in Human Visual Cortex
}

\author{
Katrin Amunts, ${ }^{1,2}$ Este Armstrong, ${ }^{1}$ Aleksandar Malikovic, ${ }^{1}$ Lars Hömke, ${ }^{1}$ Hartmut Mohlberg, ${ }^{1}$ Axel Schleicher, ${ }^{3}$ and \\ Karl Zilles ${ }^{1,3}$ \\ ${ }^{1}$ Institute of Medicine, Research Center Jülich, D-52525 Jülich, Germany, ${ }^{2}$ Department of Psychiatry and Psychotherapy, Rheinisch-Westfälische \\ Technische Hochschule, RWTH Aachen University, D-52074 Aachen, Germany, and ${ }^{3} \mathrm{C}$. and O. Vogt Institute of Brain Research, Heine University \\ Düsseldorf, D-40225 Düsseldorf, Germany
}

The structural correlates of gender differences in visuospatial processing are essentially unknown. Our quantitative analysis of the cytoarchitecture of the human primary visual cortex [V1/Brodmann area 17 (BA17)], neighboring area V2 (BA18), and the cytoarchitectonic correlate of the motion-sensitive complex $(\mathrm{V} 5 / \mathrm{MT}+/ \mathrm{hOc5})$ shows that the visual areas are sexually dimorphic and that the type of dimorphism differs among the areas. Gender differences exist in the interhemispheric asymmetry of h0c5 volumes and in the righthemispheric volumetric ratio of h0c5 to BA17, an area that projects to V5/MT+/h0c5. Asymmetry was also observed in the surface area of h0c5 but not in its cortical thickness. The differences give males potentially more space in which to process additional information, a finding consistent with superior male processing in particular visuospatial tasks, such as mental rotation. Gender differences in h0c5 exist with similar volume fractions of cell bodies, implying that, overall, the visual neural circuitry is similar in males and females.

Key words: human brain anatomy; visual system; cytoarchitecture; gender differences; asymmetry; cortical surface

\section{Introduction}

Aspects of processing visuospatial information differ between males and females. Different strategies in visuospatial navigation separate women, who typically use a landmark strategy for navigation, from men, who generally use an orientation strategy (Rahman et al., 2005). Functional magnetic resonance imaging (fMRI) also demonstrates different cortical networks in visuospatial processing: women had increased neural activity in the prefrontal and right posterior parietal cortex, whereas men showed an increased activation in the left hippocampus, right parahippocampal region, and left posterior cingulate cortex (Grön et al., 2000).

The motion-sensitive complex V5/MT+ (Zeki et al., 1991; Tootell and Taylor, 1995) also has gender differences. Potentials evoked by binocular pattern reversal, a strong stimulus to V5/ $\mathrm{MT}+$, had larger amplitudes in the right than left hemisphere in girls than in boys (Cohn et al., 1985). In an fMRI study using flickering stimuli, women had more activation than men in Brodmann area 37 (BA37), a region encompassing V5/MT+ (Kaufmann et al., 2001). A pattern reversal study showed that the P1 component of the visual-evoked potential was considerably

Received Feb. 22, 2006; revised Dec. 22, 2006; accepted Dec. 22, 2006.

This Human Brain Project/Neuroinformatics research is funded by the National Institute of Biomedical Imaging and Bioengineering, the National Institute of Neurological Disorders and Stroke, and the National Institute of Mental Health. This work was also supported by the Bundesministerium für Bildung und Forschung (BMBF) (Grant 01G00104), Brain Imaging Center West (BMBF Grant 01G00204), and the Helmholtz Association (Grant VH-NG012). We thank Ursula Blohm for excellent histological assistance.

Correspondence should be addressed to Dr. Katrin Amunts, Research Center Jülich, Institute of Medicine, 52425 Jülich, Germany. E-mail: k.amunts@fz-juelich.de.

DOI:10.1523/JNEUROSCI.4753-06.2007

Copyright $\odot 2007$ Society for Neuroscience $\quad$ 0270-6474/07/271356-09\$15.00/0 shorter in female than male infants (Malcolm et al., 2002). Central field stimulation produced a larger right than lefthemispheric response in females, whereas males had only nonsignificantly larger left hemisphere event-related potentials, suggesting a greater right-hemispheric responsiveness to moving stimuli in females (Andreassi and Juszczak, 1982).

A few studies have analyzed anatomical correlates of gender differences in visuospatial processing, including visual-motion processing. Voxel-based morphometry showed women to have a higher concentration of fibers in the right optic radiation than men (Good et al., 2001). Genders may also differ with respect to the relative size of the splenium of the corpus callosum (de Lacoste-Utamsing and Holloway, 1982; Davatzikos and Resnick, 1998), which contains interhemispheric visual fibers (Aboitiz et al., 1992), particularly V5/MT + fibers (Newsome and Allman, 1980; van Essen et al., 1981). These gender differences may indicate differences in axon number, size, or myelination, all of which could alter interhemispheric communication (Davatzikos and Resnick, 1998). Other studies have not found gender differences in absolute size (Bishop and Wahlsten, 1997; Luders et al., 2005). However, most corpus callosum studies used structural MRIs, which do not detect differences in the proportion of fibers coming from different cortical areas.

Given the observed gender differences in visual-motion perception, one would expect to find correlates in the underlying cortical microstructure. A new cytoarchitectonic area in the region of V5/MT +, hOc5 (see Fig. 1), has been identified recently (Malikovic et al., 2007) as a plausible anatomical candidate for human V5/MT+ (Wilms et al., 2005; Barnikol et al., 2006). In this study, we analyzed the volumes and the volume fraction of cell bodies in hOC5 (V5/MT+) and compared them with those 

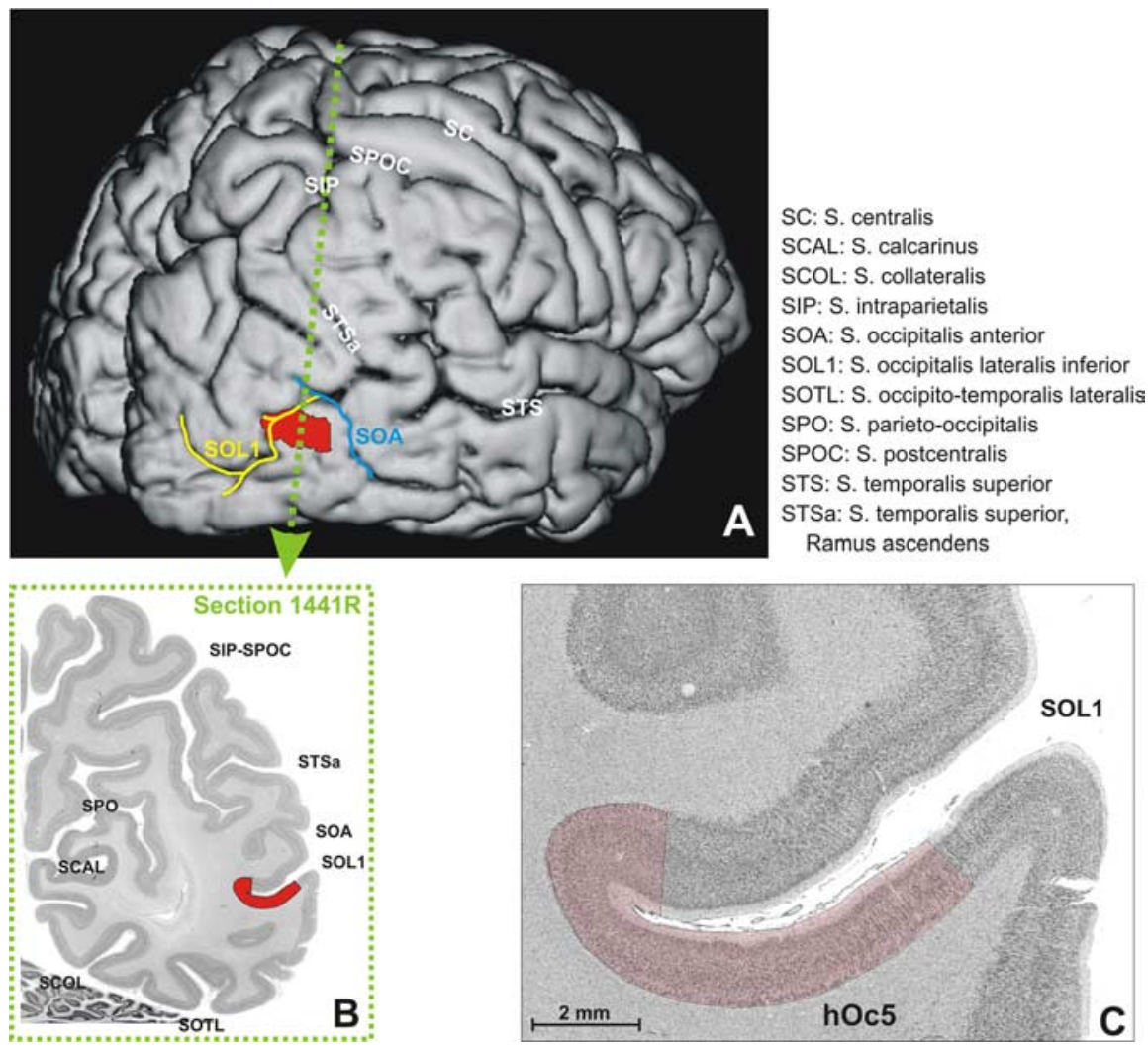

Figure 1. A, Surface rendering of a 3D-reconstructed dataset of a human brain (female, 72 years of age). Right hemisphere, posterolateral view. h0c5 (red) was projected to the free surface of the brain. The area is mainly hidden in the depths of two sulci; the anterior occipital sulcus (aqua) and the inferior lateral occipital sulcus (yellow). The dotted line marks the approximate location of the histological section shown in B. B. The location of h0c5 is labeled in red. C, Cytoarchitecture of area h0c5 (red transparency) in the histological section at higher magnification.

from BA17 and BA18. Because of the retinotopic organization of the visual cortex, we also calculated the surfaces areas of BA17, BA18, and hOc5 and analyzed those with respect to volume, interhemispheric, and gender differences.

\section{Materials and Methods}

Although Brodmann's cytoarchitectonic map is oversimplified (Brodmann, 1909), and new ones have been published (Clarke, 1993; Amunts et al., 2000; Annese et al., 2005; Malikovic et al., 2007; Rottschy et al., 2007), his nomenclature serves as a general reference. Our study analyzes BA17 and BA18, as well as the recently found microstructural correlate of the motion-sensitive complex V5/MT+, area hOc5, part of BA19. Areas hOc5, BA17, and BA18 were studied in cell-body-stained, serial sections (thickness, $0.02 \mathrm{~mm}$ ) from 10 human postmortem brains, five males and five females (Amunts et al., 2000) (Fig. 1). The mean age of the subjects was 66.3 years and ranged from 37 to 85 years; the postmortem delay was $<36$ h (Table 1). Brains were obtained from the body donor program of the Institute of Anatomy at the University of Düsseldorf (Düsseldorf, Germany), in accordance with the guidelines of the Ethics Committee of the University of Düsseldorf. They were histologically processed and three-dimensionally (3D) reconstructed as described previously (Amunts et al., 2000; Zilles et al., 2002).

The cortical regions were delineated by determining significant differences in the gray level index (GLI) (Schleicher and Zilles, 1990) (Figs. 2, 3 ). The GLI, the proportion of cell bodies per unit brain tissue, was collected from profiles made perpendicular to the pial surface using an image analysis system (KS 400; Zeiss, Oberkochen, Germany). Profiles, therefore, capture the cytoarchitecture of a cortical area. Ten features defined the shape of each profile and form the basis for observerindependent definition of area borders (Schleicher et al., 1999; Amunts et al., 2000). Cytoarchitectonic borders were located where a multivariate distance measure (Mahalanobis distance) and a subsequent Hotellings $T^{2}$ test showed a significant difference between profiles in shape, thus making the borders of hOc5, BA17, and BA18 objective (Schleicher et al., 1999). All analyses used cortical parcellations of the same sample of postmortem brains and have been described in detail previously (Amunts et al., 2000; Malikovic et al., 2007).

Cortical volumetrics. The cortical areas were labeled in high-resolution images (resolution of the digitized images, $7000 \times 6000$ pixels) of histological sections using in-house software (Fig. 4). The histological sections are $1.2 \mathrm{~mm}$ apart (each 60th section). The volumes of the structures (Table 1) were calculated from area measurements in the 3D-reconstructed histological sections using the following formula: $V=s \times T \times x \times y \times \Sigma A_{\mathrm{i}} \times F$, where $V$ is the volume of the cortical region (result in cubic millimeters), $s$ is the distance between two measured sections (number of sections), $T$ is the thickness of a histological section $(0.020 \mathrm{~mm})$ $x$ is the width of a pixel $(0.02116 \mathrm{~mm}), y$ is height of a pixel $(0.02116 \mathrm{~mm}), \Sigma A_{\mathrm{i}}$ is the sum of areas of the structure over all sections (in pixels), and $F$ is the shrinkage factor of each individual brain.

Approximately 20 sections were analyzed per area, hemisphere, and brain. To estimate the true volumes, shrinkage from histological processing was taken into account by calculating individual shrinkage factors for each brain ( $\mathrm{Ta}$ ble 1) (Amunts et al., 2005). Shrinkage differs with respect to age, sex, clinical history, brain size, cause of death, autopsy conditions, histological techniques, and other factors (Vierordt, 1893; Blinkov and Glezer, 1968; Haug, 1980; Skullerud, 1985). Volumes of hOc5, BA17, and BA18 were analyzed statistically using an ANOVA with repeated-measures design (within factors, area and hemisphere; between factor, gender). A significance level of $\alpha=0.05$ was set for all tests. Age was used as a covariate.

The volumetric ratios of hOc5 to BA17 and to BA18 were calculated for each hemisphere and separately for males and females. These ratios exclude effects related to differences in the absolute brain size between males and females. The ratios were analyzed using an ANOVA [within factors, pair of area (hOc5 and BA17, hOc5 and BA18) and hemisphere; between factor, gender] for main effects and interactions.

Volume fractions of cell bodies. Analyses on gender, inter-area, and interhemispheric differences in the volume fractions of cell bodies were based on mean GLI values calculated for areas hOc5, BA17, and BA18. The mean values came from GLIs measured in $10-20$ profiles in each histological section and from at least three sections per area, hemisphere, and brain. This approach ensured that profiles were collected from similar parts of the cortex (i.e., from the wall of gyri in which geometrical distortions attributable to the sectioning plane are minimal). An ANOVA with a repeated-measure design (within factors, area and hemisphere; between factor, gender) checked for significance (significance level of $\alpha=0.05$ ).

Surface areas and cortical thickness. The present study extracts surface measurements from three-dimensional reconstructions of delineated cortical areas. Although several powerful tools exist to analyze cortical surfaces of whole hemispheres of the living human brain (Fischl et al., 1999; van Essen et al., 1998, 2001), such methods cannot be directly applied to individual cortical areas delineated in histological sections of postmortem brains. In contrast to whole hemispheres, which have closed contours, areas defined in histological sections have two open contours: 
Table 1. Postmortem sample, fresh brain weights (in grams), and volumes of areas (V, $\mathrm{mm}^{3}$ )

\begin{tabular}{|c|c|c|c|c|c|c|c|c|c|}
\hline Age (years) & Sex & Fresh brain weight & Shrinkage correction $f_{\mathrm{v}}$ & V(BA17) left & V(BA17) right & V(BA18) left & $\mathrm{V}$ (BA18) right & V(h0c5) left & V(h0c5) right \\
\hline 37 & $\mathrm{~m}$ & 1437 & 2.02 & 9423 & 9207 & 4134 & 4405 & 357 & 648 \\
\hline 54 & $\mathrm{~m}$ & 1757 & 2.45 & 10437 & 11052 & 5385 & 5900 & 591 & 1160 \\
\hline 56 & $\mathrm{~m}$ & 1270 & 2.20 & 7518 & 7160 & 6698 & 6999 & 244 & 391 \\
\hline 75 & $\mathrm{~m}$ & 1349 & 2.15 & 8528 & 8667 & 6369 & 7140 & 475 & 772 \\
\hline 69 & $\mathrm{~m}$ & 1360 & 2.13 & 9219 & 9000 & 7723 & 8418 & 483 & 601 \\
\hline 85 & $f$ & 1046 & 1.67 & 5302 & 5359 & 4520 & 5266 & 422 & 266 \\
\hline 59 & $f$ & 1142 & 2.06 & 6025 & 6035 & 6194 & 6517 & 228 & 283 \\
\hline 79 & $f$ & 1350 & 1.93 & 7616 & 6872 & 6718 & 6269 & 330 & 195 \\
\hline 72 & $f$ & 1216 & 1.82 & 5425 & 5820 & 4847 & 3239 & 302 & 267 \\
\hline 79 & $f$ & 1110 & 1.47 & 7040 & 6735 & 4054 & 4709 & 323 & 356 \\
\hline Mean & & & 1.99 & 7653 & 7591 & 5664 & 5886 & 376 & 494 \\
\hline SD & & & 0.28 & 1752 & 1817 & 1255 & 1518 & 115 & 302 \\
\hline
\end{tabular}

Mean fresh brain weight of females (f) was $1137 \mathrm{~g}$ and that of males (m) was $1390 \mathrm{~g}$.
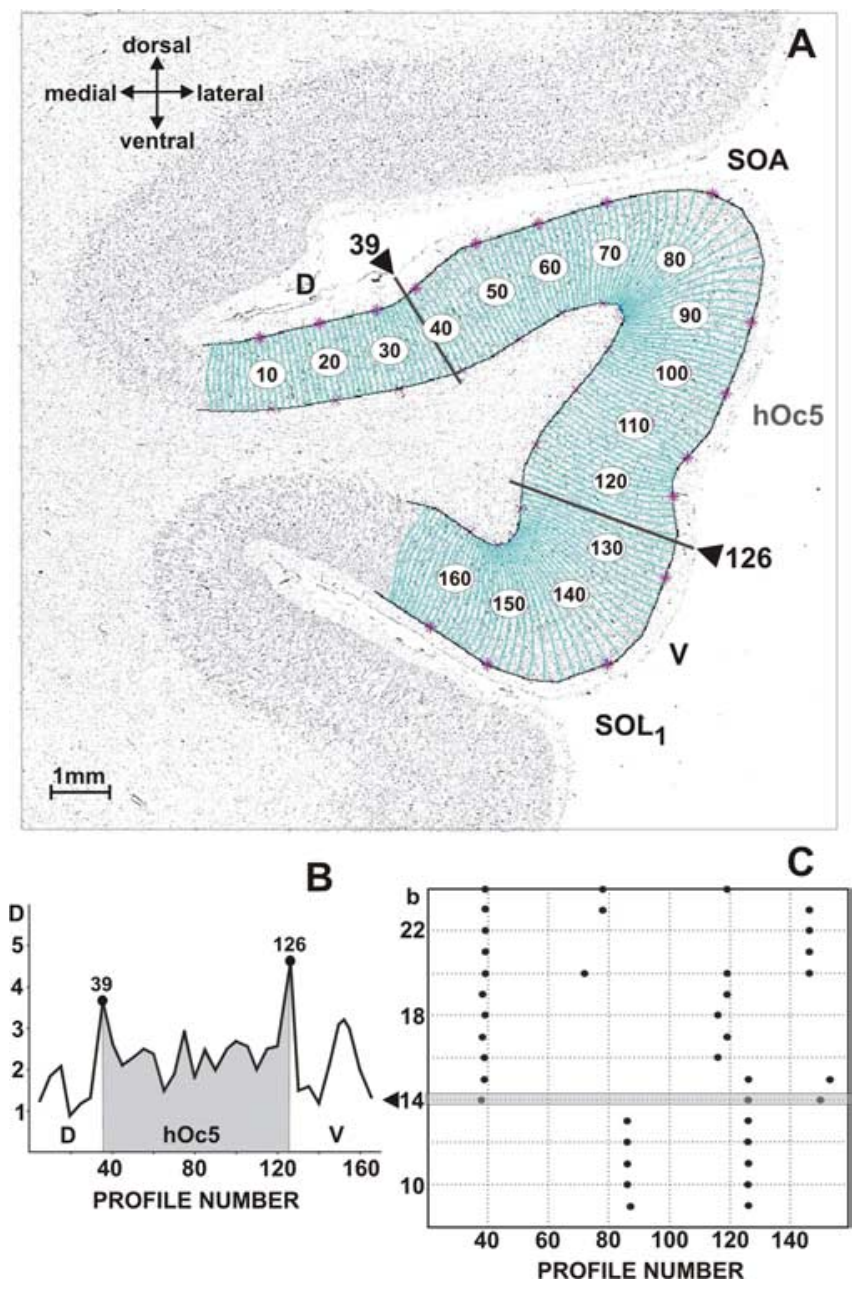

Figure 2. A, Definition of cortical borders of h0c5 in a cell-body-stained histological section of a postmortem brain using an observer-independent method (Schleicher et al., 1999). GLI image of a coronal section from the region of the anterior occipital sulcus (SOA) and the inferior lateral occipital sulcus $\left(\mathrm{SOL}_{1}\right)$. Arrowheads and lines indicate the borders between area h0c5 and the neighboring dorsal (D) and ventral (V) areas. The cortex is covered from the layer I/II border to the layer $\mathrm{VI} /$ white matter border by a series of traverses. Along these traverses, profiles, which capture laminar changes of the GLI (i.e., the volume fraction of cell bodies) from the surface to the white matter, i.e., the cytoarchitecture, were extracted. Profiles are numbered from 1 to 169 . $\boldsymbol{B}$, Multivariate distance $D$ (Mahalanobis distance) as a measure of similarity of neighboring blocks of profiles in dependence on the profile number. Block size $b=14$ profiles. Significant peaks of $D$ indicate positions of cytoarchitectonic borders. They were found at positions 39 (dorsal border) and 126 (ventral border). C, The distance D was calculated for different block sizes ranging from $b=8$ to 24. The distance for $b=14$ is highlighted.

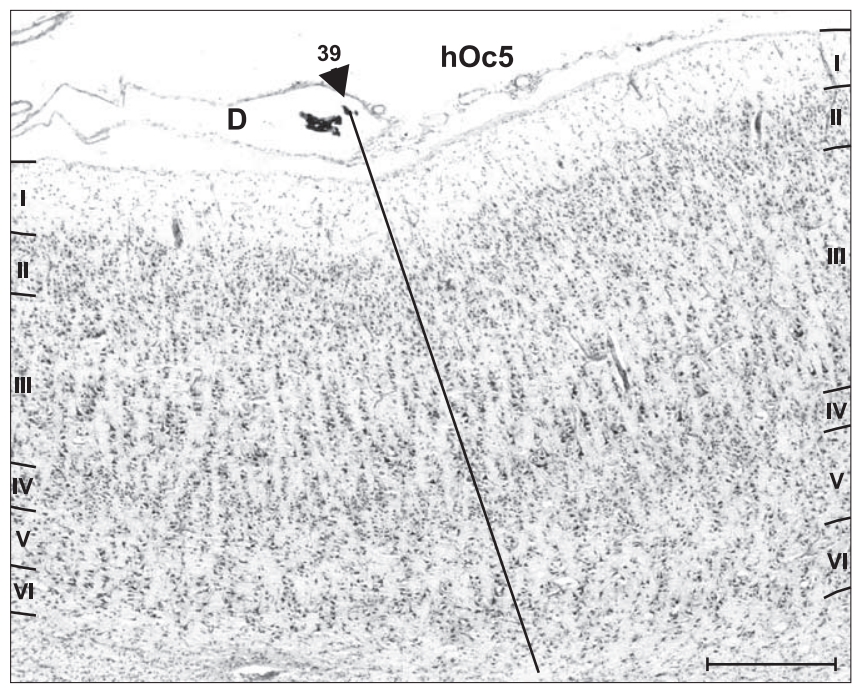

Figure 3. Dorsal border of area h0c5 with area D. The sizes of pyramidal cells in sublayer Illlc are larger in area h0c5 than in area D. Roman numerals indicate cortical layers. Scale bar, 0.5 $\mathrm{mm}$. Same border as in Figure 2 at position 39 .

the outer (pial) and inner (white matter) boundaries. Many methods are not suitable for processing datasets containing open contour lines (e.g., NUAGE software) (Geiger, 1993).

Furthermore, generating topologically correct representations of sliced objects when the distances between slices are much greater than the within-plane resolution is not trivial (Boissonnat, 1988; Meyers et al., 1991; Meyers, 1994). The difficulties are compounded when the number of contour lines in consecutive sections differs, the reconstructed surface patch is not closed, or it ramifies into multiple endings in different sections. These difficulties confront researchers analyzing cortical areas. Additionally, in most previous approaches, adjacent layers were assumed to be independent, and, thus, the process of reconstruction merged interpolated models derived from pairs of successive parallel slices (Barequet et al., 2004). This assumption may result in frequent topological defects for highly folded surfaces, like cortical areas. Finally, the data modalities, spatial resolution of the images, and image artifacts are also different in histological sections of postmortem brains compared with in vivo MR data.

Therefore, for the present study, we adapted and extended existing methods to measure surfaces. Before beginning the triangulation process, we fixed topological problems using a Reeb graph-based representation of the topology (Reeb, 1946; Biasotti, 2004, 2005). For each contour lying in the $x-y$ plane of section $z_{\mathrm{i}}$, we computed a bounding box with a height $0.5\left(z_{\mathrm{i}+1}-z_{\mathrm{i}-1}\right)$. Next, we enlarged the height of each bounding box until it overlapped those of its neighbors and adequately represented the surface.

After this, two consecutive contour lines, one from each edge, were 

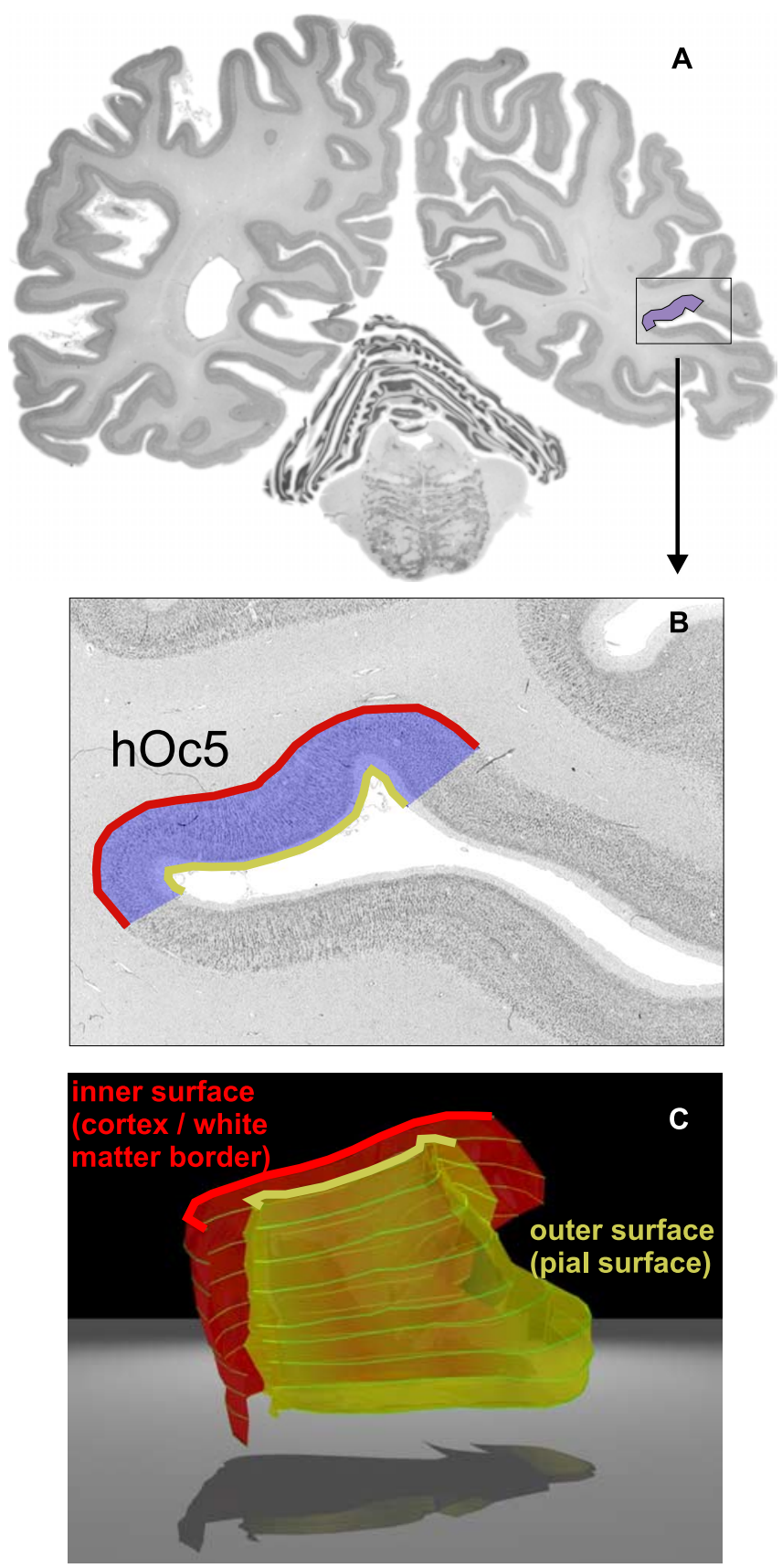

Figure 4. Definition of the surfaces of area $\mathrm{h} 0 \mathrm{c} 5$ in a 3D-reconstructed postmortem brain. $\boldsymbol{A}_{\boldsymbol{t}}$ Label of the area in a histological section. $\boldsymbol{B}$, Region of interest of $A$ but at higher magnification. Bold lines mark the outer (dark yellow) and inner (red) contours of the area. $C$, Surface reconstruction of the area, view from the sulcus. The approximate positions of the histological sections, in which $\mathrm{h} 0 \mathrm{c} 5$ was delineated, are indicated by green lines. The topmost line corresponds to the section shown in $\boldsymbol{A}$.

automatically triangulated. Triangles were built so that they did not intersect any existing triangle, and the lengths of its edges were as similar as possible. The quality of individual triangulations was checked by comparing actual values with the mean regularity value $\sigma$, defined as the ratio between the inner and outer circle radius, where $\sigma_{\text {best }}=0.5$ for an equilateral triangle. Triangles closest to the optimal value were processed further. As a result of the larger interslice distance than in-plane resolution and the local heuristic tiling, the approach produced many relatively small triangles.

To get a smooth surface without significantly altering the distance between the inner and outer surfaces, we implemented a Laplace mesh
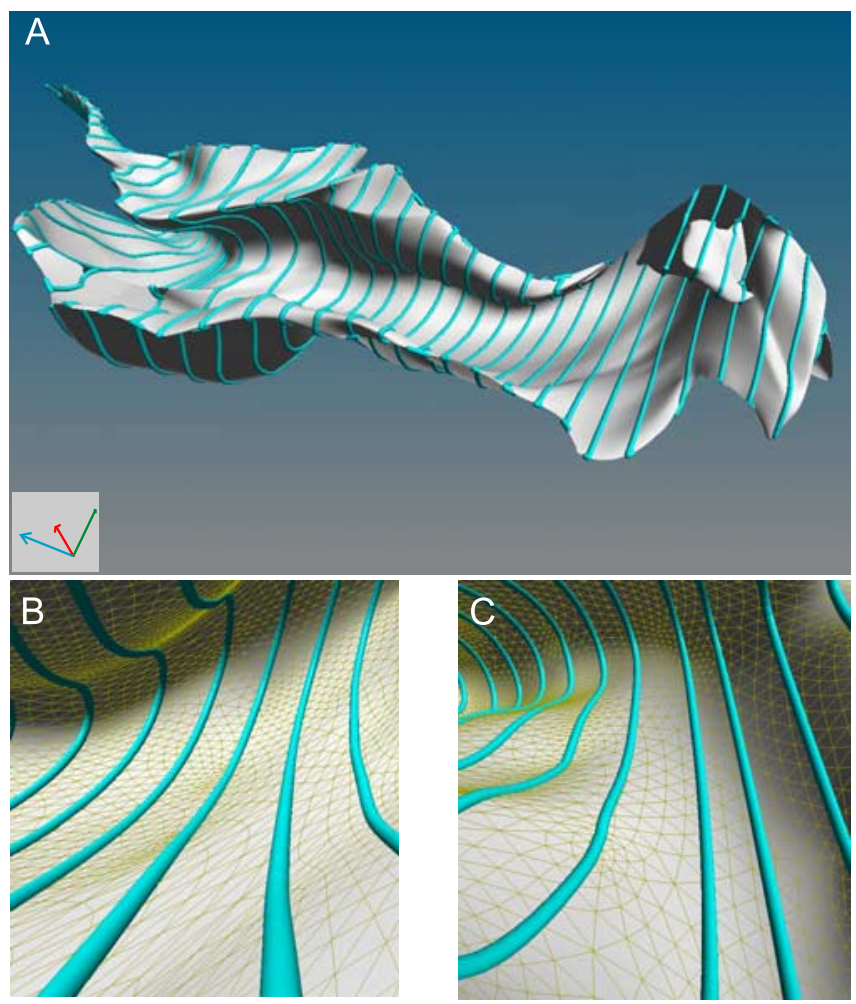

Figure 5. Reconstruction of the inner (cortex-white matter) surface of BA17 in serial histological sections of a left hemisphere. View into the calcarine sulcus. $A$, The sequence of contours of the delineated area ("tubes") in histological sections is projected to the surface. The distance between histological sections is $1.2 \mathrm{~mm}$ (distance between tubes). The calcarine sulcus can be identified as the elongated concave structure, which is surrounded by the "U-shaped" area. $\boldsymbol{B}$ and ( show two regions with triangulation (yellow triangles) at higher magnification. Note the regular shape of the triangles. A total of 200,000 triangles have been generated in this particular area to create its surface. The arrow cross shows the orientation within the brain: green arrow, dorsal/ventral; blue arrow, caudal/rostral; red arrow, medial/lateral.

smoothing method, which enabled relatively large movements of vertices along the original contour line but prevented movement perpendicular to the surface. Next, a global mesh refinement, which divided each triangle into four smaller triangles, was applied. Afterward, we flipped all nonlocally Delaunay edges to be locally (O’Rourke, 1998) [vTK software package (http://public.kitware.com/VTK/get-software.php)]. The surface was smoothed again with the same Laplace smoothing method described above except that minor movements perpendicular to the surface were allowed. Finally, we checked and manually corrected the triangulation in places in which the topology determined by the algorithm was obviously erroneous. That was the case in some parts of area V1/BA17, in which the structure is highly complex (Fig. 5).

The quality of the total triangulation, like that of individual triangles, was checked by calculating the mean of the regularity factor $\sigma: \sigma_{\mathrm{V} 1 / \mathrm{BA} 17}=$ $0.408 \pm 0.009, \sigma_{\mathrm{V} 2 / \mathrm{BA} 18}=0.392 \pm 0.013$, and $\sigma_{\mathrm{hOc} 5}=0.401 \pm 0.017$. The small SDs indicate a regular triangulation, whereas differences of mean values $\sigma$, from $\sigma_{\text {best }}$, reflect the relatively large interslice distance, which could not be totally removed.

The contour-labeled areas served as a basis for subsequent brain morphometry. Surface areas were analyzed using ANOVA with repeatedmeasures design as described above. Areas were corrected for shrinkage by using the same shrinkage factor determined for the volume of the brain, $f$, raised to the two-thirds power $\left(f^{2 / 3}\right)$. We assumed an isometric shrinkage in all three spatial directions.

To estimate the mean cortical thickness of the areas, the area volume was divided by its mean surface, which was calculated as the average of the outer (pial) and the inner (cortex/white matter border); additional information is shown in the supplemental data (available at www.jneu- 

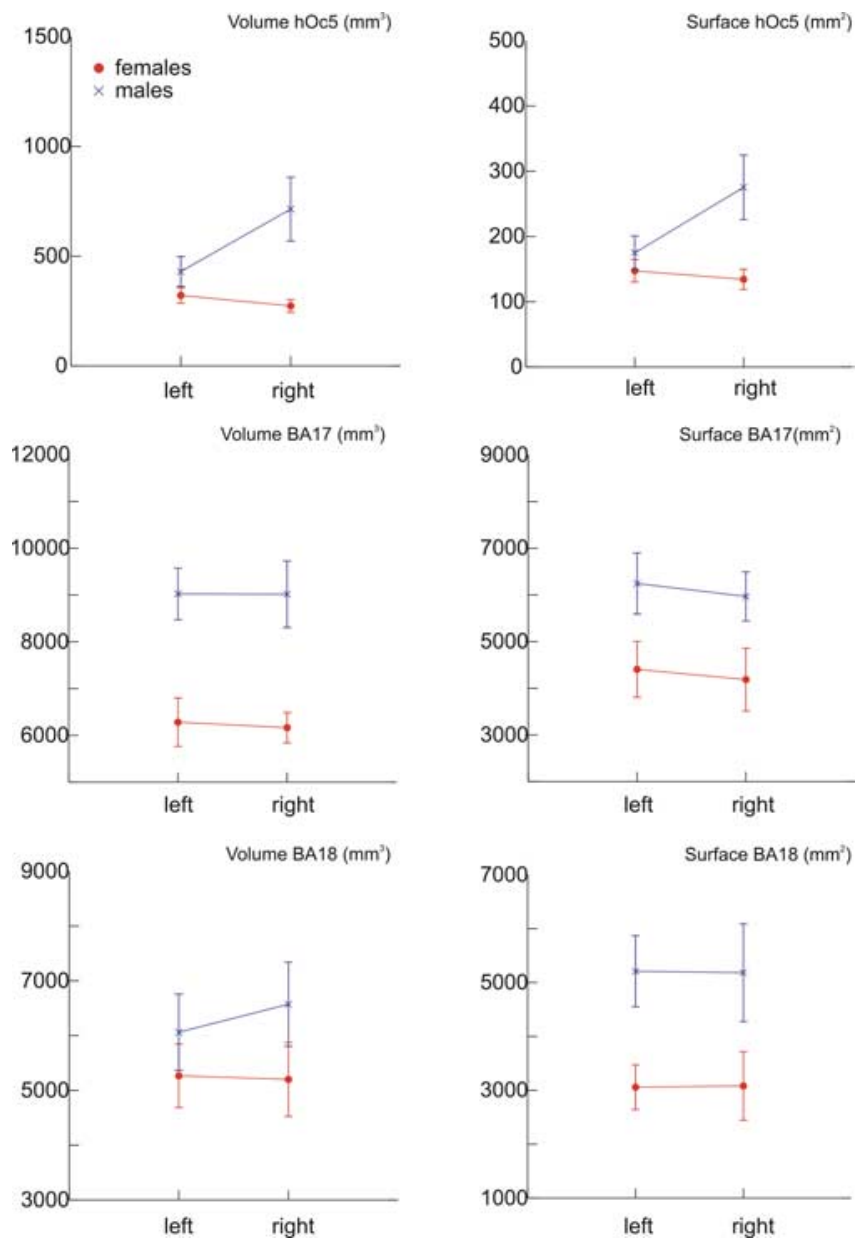

Figure 6. Mean \pm SE volumes and surface areas of areas h0c5 (top row), BA17 (middle row), and BA18 (bottom row) of right and left hemispheres, in males and females. The amount and direction of the bilateral asymmetry of h0c5 differs between genders $(p<0.05)$. Whereas males showed a significant right-larger-than-left asymmetry in volume and surface of h0c5, females were more symmetrical.

rosci.org as supplemental material). The mean cortical thickness was corrected for shrinkage with $f^{1 / 3}$.

\section{Results}

Previous structure/function studies determined that V5/MT+ overlaps and is coterminous with the cytoarchitectonically defined area hOc5 (Wilms et al., 2005; Barnikol et al., 2006). A distinct microstructure separates area hOc5 from neighboring extrastriate areas; it includes a broader layer III, a higher cell density in layers II/III, and a lower one in layer V (Fig. 3). Most of hOc5 was found deep in the anterior part of the inferior lateral occipital sulcus or in the anterior occipital sulcus (Fig. 1). The cytoarchitectonic details of hOc5 (Malikovic et al., 2007) as well as of BA17 and BA18 (Amunts et al., 2000) have been published previously.

The ANOVA of pooled gender data showed a significant volumetric difference between males and females $(p<0.01)$ and a significant area by gender interaction $(p<0.05$ ) (Fig. 6, Tables 1 , $2)$. Subsequent univariate $F$ tests showed significant size differences between males and females in left and right BA17, as well as in the right hOc5 $(p<0.01)$, with the right hOc5 being significantly larger in male than female brains (Fig. 6). Moreover, three of five female brains had smaller right than left hOc5 volumes, whereas all of the male brains of this study had larger right than left hOc5 volumes (Table 1). Although the mean size of male brains in this study was larger than that of female brains (by a factor of 1.2), the difference between the genders in the right hOc5 volume (factor of 2.6) was considerably greater than expected on the basis of brain size. The gender difference in the left hOc5 volume resembles that of brain size (factor of 1.3).

Given that BA17 and BA18 interconnect with V5/MT+, we next determined whether or not earlier node(s) in the serial processing of visual information were also asymmetric: is asymmetry observed in beginning stages of visual processing, in BA17, and/or does it appear only later, in higher associative visual areas, i.e., in BA18 or hOc5 (V5/MT+)? The data showed that both BA17 and BA18 were bilaterally symmetric with respect to volume $(p>0.05)$ (Table 1). However, the volume of BA17 differed between males and females, with males having larger volumes than females on both sides (Fig. 6) $(p<0.05)$. The volume of right BA18 was larger than the left in all male brains but only in three female brains; this difference did not reach significance (Fig. 6, Table 1). The observed gender-related interhemispheric asymmetry of hOc5 in volume is therefore not a feature of the entire visual cortex or even the whole brain but a specific aspect of the anatomy of hOc5.

The volumetric ratio of hOc5-to-BA17 was significantly smaller in the female right hemisphere than it was in males $(p<$ 0.05 ) (Fig. 7). The right hOc5-to-BA18 ratio only approached significance $(p=0.06)$. Both ratios were approximately twice as small in female brains as they are in male brains. Thus, females had more primary and secondary visual cortex per volume unit hOc5 in the right hemisphere than do males. Age effect was not a significant factor in any of the parameters $(p>0.05)$.

To examine whether and how the observed quantitative shifts in the volumes of hOc5, BA17, and BA18 affected the underlying cytoarchitecture, we analyzed the volume fraction of regions of cell bodies. A decreased volume fraction of cell bodies is equivalent to an increased proportion of neuropil, i.e., more space between cell bodies that is occupied by dendrites, axons, and synapses. To begin answering this question, we averaged the GLI, the proportion of cell bodies per unit brain tissue, across all cell layers and compared the mean GLI between the two hemispheres and genders (Fig. 8).

At this level, GLIs were not sexually dimorphic. Instead, the GLIs of hOc5, BA17, and BA18 were similar in having a small but significant right-larger-than-left asymmetry $(p<0.05)$. The lower GLI values of the left hemispheres indicate that all three visual areas have more neuropil than the structures do in the right hemispheres.

Differences in the volume of hOc5 between males and females can be caused on a macroscopic level by two factors: differences in the surface of the areas and/or differences in cortical thickness. Surface measures of cortical areas are highly relevant parameters considering the retinotopic organization of visual areas (Huk et al., 2002).

Areas, like volumes, have similar patterns of male-female differences (Fig. 6, Table 2). Male and female surface areas differed significantly between each other $(p<0.001)$, and there was a significant gender by area interaction $(p<0.05)$. Right area hOc5 was significantly smaller in female than in male brains, whereas left hOc5 did not differ between genders (Fig. 6). Significant differences between males and females were found in the left and right BA17 as well as in left BA18, with males having larger surface areas than females; the latter differences, however, did not interact with hemisphere. The ratios of all left- 
Table 2. Postmortem sample, surfaces of areas $\left(A, \mathrm{~mm}^{2}\right)$

\begin{tabular}{|c|c|c|c|c|c|c|c|c|c|}
\hline Age (years) & Sex & Fresh brain weight $(\mathrm{g})$ & Shrinkage correction $f_{\mathrm{A}}=f_{\mathrm{V}}{ }^{2 / 3}$ & $A(B A 17)$ left & $\mathrm{A}(\mathrm{BA} 17)$ right & $A(B A 18)$ left & $\mathrm{A}(\mathrm{BA} 18)$ right & $A(h 0 c 5)$ left & $\mathrm{A}(\mathrm{h} 0 \mathrm{c} 5)$ right \\
\hline 37 & $\mathrm{~m}$ & 1437 & 1.60 & 8001 & 7416 & 3395 & 2206 & 146 & 278 \\
\hline 54 & $\mathrm{~m}$ & 1757 & 1.82 & 6240 & 5032 & 4329 & 5285 & 209 & 424 \\
\hline 56 & $\mathrm{~m}$ & 1270 & 1.69 & 4373 & 4934 & 6457 & 5328 & 101 & 167 \\
\hline 75 & $\mathrm{~m}$ & 1349 & 1.67 & 6356 & 6109 & 5801 & 6524 & 227 & 292 \\
\hline 69 & $\mathrm{~m}$ & 1360 & 1.65 & 6255 & 6349 & 6061 & 6566 & 192 & 215 \\
\hline 85 & $f$ & 1046 & 1.41 & 3825 & 3875 & 2468 & 2136 & 188 & 101 \\
\hline 59 & $f$ & 1142 & 1.62 & 4414 & 5449 & 4219 & 4964 & 98 & 131 \\
\hline 79 & $f$ & 1350 & 1.55 & 6425 & 5675 & 3573 & 3433 & 154 & 135 \\
\hline 72 & $f$ & 1216 & 1.49 & 3759 & 2798 & 2734 & 1809 & 134 & 121 \\
\hline 79 & $f$ & 1110 & 1.29 & 3602 & 3136 & 2297 & 3067 & 162 & 183 \\
\hline Mean & & & 1.58 & 5325 & 5077 & 4134 & 4132 & 161 & 205 \\
\hline SD & & & 0.15 & 1511 & 1456 & 1524 & 1820 & 43 & 100 \\
\hline
\end{tabular}

m, Male; f, female.
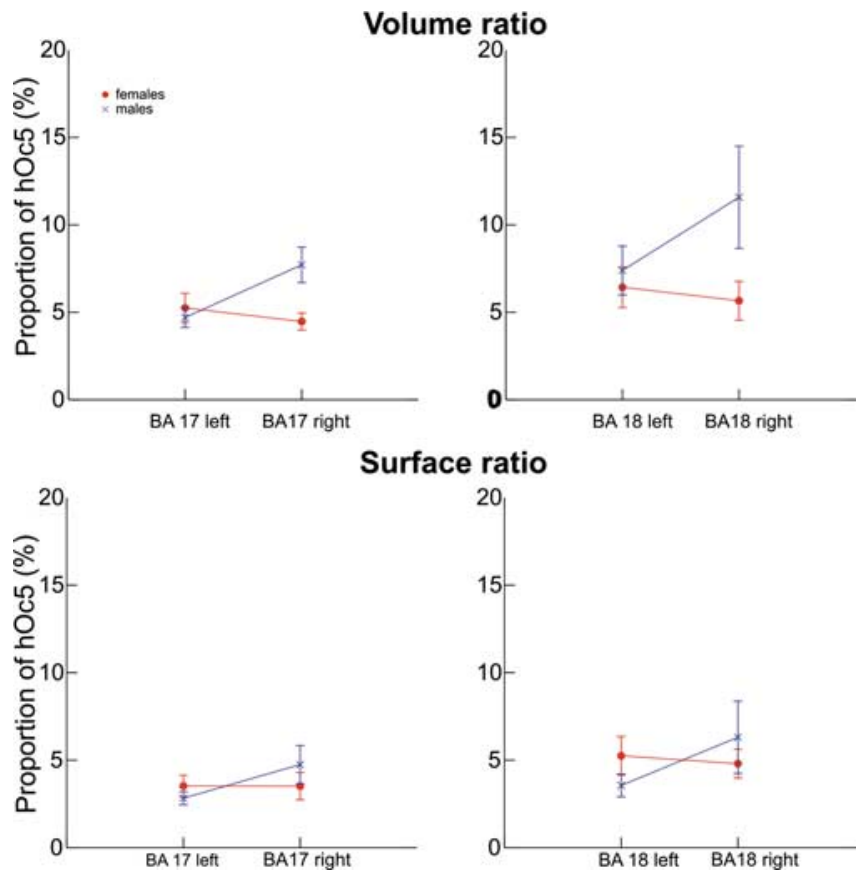

Figure 7. Ratios (in percentage, $\pm \mathrm{SE}$ ) between volumes of h0c5-to-BA17 and h0c5-toBA18 (top row) show a significant gender difference for right h0c5-to-BA17 $(p<0.05)$ and a similar but nonsignificant tendency for right h0c5-to-BA18 ( $p=0.06)$. In the right hemisphere, the female ratios were approximately twice as small as they are in male brains (for both h0c5-to-BA17 and h0c5-to-BA18). Ratios did not differ between each other in the left hemisphere. Ratios between surfaces of the areas (bottom row) show similar directions of asymmetry $(p>0.05)$.

hemispheric combinations of areas did not differ significantly between males and females (Fig. 7) (all $p>0.05$ ).

In contrast to volume and surface area, cortical thickness is not an independent parameter with patterns that differ from those of volumes or surface areas (Fig. 9). Cortical thickness seems to differ between males and females in hOc5, with males having thicker cortices than females in both hemispheres. The opposite was found in BA18, with females having thicker cortices than males. Thickness was generally greater in hOc5 than in both BA17 and BA18 (supplemental data, available at www.jneurosci. org as supplemental material).

\section{Discussion}

\section{Methodical considerations}

The present morphometric study is based on (1) an objective definition of borders between cytoarchitectonically defined cor-

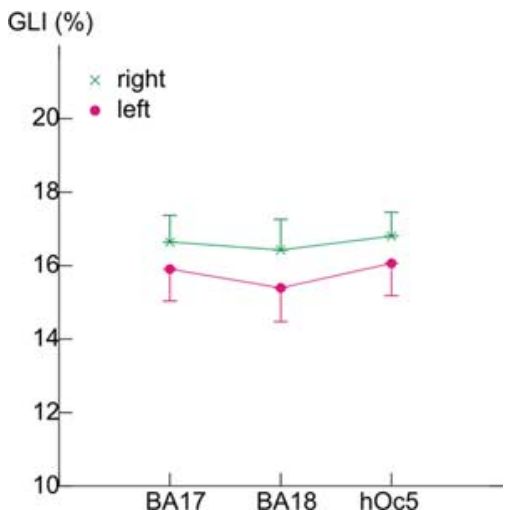

Figure 8. Volume fraction of cell bodies (GLI $\pm \mathrm{SE}$ ) has significantly higher values on the right than left side, in all three areas, regardless of gender. The larger GLI values on the right mean that there are more cell bodies and less room for neuropil; dendrites, axons, synapses.

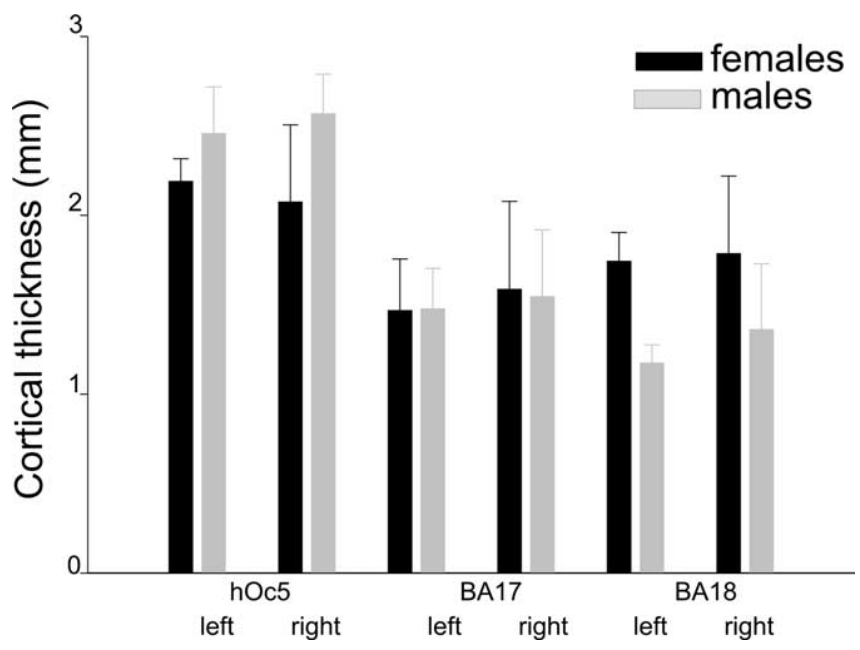

Figure 9. Mean $\pm S D$ cortical thicknesses of areas $\mathrm{h} 0 \mathrm{c} 5, \mathrm{BA} 17$, and $\mathrm{BA} 18$ for the left and the right hemisphere, separately for males $(n=5)$ and females $(n=5)$. Note that the thickness is smaller in BA17 plus BA18 than in h0c5. Females have a thicker cortex than males in BA18, whereas males have a thicker $\mathrm{h} 0 \mathrm{c} 5$ cortex than females.

tical areas, (2) the calculation of volumes using a stereological formula, and (3) the calculation of areal surfaces based on threedimensional reconstructions of the areas. Whereas the first two steps have been proven in many studies, the third step represents a new methodological development (for comparison of area estimates of the literature and that of the present study, see supplemental data, available at www.jneurosci.org as supplemental 
material). The present study provides, for the first time, threedimensional surfaces of microstructurally defined areas of the visual cortex. Such measurements represent a methodical advantage compared with measurements from serial sections, which systematically underestimate the true surface (Fahle and Palm, 1983).

With surfaces defined in three dimension, it is possible to estimate, in a first approach, the mean cortical thicknesses for each area. Because the thickness value is derived from volumes and surfaces, it is not an independent parameter and was not analyzed statistically. The advantage of this way to estimate the cortical thickness is the robustness of the parameter. Thickness provides a measure to test the plausibility of areal surfaces (supplemental data, available at www.jneurosci.org as supplemental material).

The estimated thicknesses coincide well with the data provided by von Economo and Koskinas, who reported that the calcarine cortex is $\sim 2 \mathrm{~mm}$ thick in the convexities and $1.2 \mathrm{~mm}$ thick in the bank of the sulcus. According to their Figures 28 and 29 (von Economo and Koskinas, 1925), cortical thickness in the region approximating hOc5 ranges from 2.7 to $2.9 \mathrm{~mm}$ (supplemental data, available at www.jneurosci.org as supplemental material).

The volumes of cortical regions can be estimated with high precision in serial sections using Cavalieri's principle (Uylings et al., 1986). The sample size of the present study is relatively low compared with MR studies in the living human brain (Macdonald et al., 2000; Kovalev et al., 2003; Lerch and Evans, 2005). Advantages of the present approach, however, include the high spatial resolution and, therefore, precise measurements, as well as associating the latter with cytoarchitectonically defined areas instead of with brain macroscopy. Finally, it has to be mentioned that a systematic cytoarchitectonical analysis and mapping study as presented here is extremely time consuming and labor intensive. Such constraints limited the size of the sample, which was subjected to detailed brain morphometry. As a consequence, we may assume that certain gender, age, areal, and/or interhemispheric differences did not reach significance because of a considerable amount of intersubject variability.

\section{Gender-related differences in visual areas}

As described above, gender differences in the visual cortices may link to well described brain functions. V5/MT+ is part of the dorsal stream ("where" system), i.e., the occipitoparietal pathway (Ungerleider and Mishkin, 1982; Ungerleider and Haxby, 1994). It is widely accepted that V5/MT+ makes important contributions to a number of aspects of motion perception (Schenk et al., 2005). V5/MT+ receives projections from areas V1 (BA17) and V2 (BA18) (van Essen et al., 1981, 1986; Maunsell and van Essen, 1983; Shipp and Zeki, 1989; Fellemann and van Essen, 1991) and from subcortical regions, e.g., the lateral geniculate body and the pulvinar (Yoshida and Benevento, 1981; Rodman et al., 1990; Shipp, 2001; Sincich et al., 2004). It sends feedback projections to the primary visual cortex (Pascual-Leone and Walsh, 2001; Sillito and Jones, 2002; Muckli et al., 2005; Silvanto et al., 2005). As part of the dorsal stream, V5/MT+ projects to VIP and LIP in the cortex of nonhuman primates, areas that are located in the intraparietal sulcus (Maunsell and van Essen, 1983; Fellemann and van Essen, 1991; Orban, 1997; Lewis and van Essen, 2000).

Several lines of evidence support the notion that area hOc5 is the cytoarchitectonic correlate of functionally defined V5/MT+: (1) an observer-independent method has identified and mapped a distinct microstructure (Malikovic et al., 2006), which (2) co- incides with the stereotaxic location and sulcal landmarks of an area whose functional activities characterize V5/MT+ (Wilms et al., 2005; Barnikol et al., 2006) and to (3) the overlapping locales of an in vivo observer-independent high-resolution MR map and fMRI of V5/MT + (Walters et al., 2007). The location of hOc5 on the posterior bank of the anterior occipital sulcus (Malikovic et al., 2007) also makes it unlikely to be part of MT or MST (Huk et al., 2002),

Morphometric differences in the cytoarchitecture of hOc5, BA17, and BA18 may underlie observed gender differences in processing of visuospatial and motion information. Considering that $\mathrm{V} 5 / \mathrm{MT}+$ has been characterized as an area, which combines information about space, integrates V1 input, and, under some conditions, integrates them to compute pattern motion (Born and Bradley, 2005), we may speculate that the sexually dimorphic differences in the ratios of primary and secondary visual cortices to hOc5/V5/MT + suggest a stronger convergence of visual information from BA17/BA18 to the right hOc5 in females than in males. The increased amplitudes and activations in right female hemispheres noted above accord with that interpretation (Andreassi and Juszczak, 1982; Cohn et al., 1985; Kaufmann et al., 2001). Alternatively, smaller female hOc5 volumes may reflect a sparser sampling of BA17/BA18 by the right hOc 5 of women than of men. This alternative, however, seems less plausible; more activation has been observed in female BA37 than in male (Kaufmann et al., 2001).

Right-hemispheric dominance in a mental rotation task, which was independent of the response hand, was observed in males, but not females, and was interpreted as reflecting a more bilateral organization of visuospatial processing in females than in males (Johnson et al., 2002). At a structural level, an MRI texture analysis of 290 subjects found males to be more asymmetric in the majority of brain regions, including the presumed region of V5/MT + (Kovalev et al., 2003). Volumetric analyses in a sample of postmortem fetal brains in the Yakovlev Collection showed that male striate-extrastriate cortices were far more asymmetrical than they were in female brains (de Lacoste et al., 1991). Another study failed to demonstrate a significant gender difference but did find a significant right-larger-than-left volumetric asymmetry of the human striate cortex (Murphy et al., 1996). Summarizing, most studies, including ours, find that female visual structures are more bilaterally symmetric. Furthermore, male asymmetry in mental rotation tasks accords with our findings: a larger right male V5/MT + has more space in which to process information from regions outside BA17/BA18.

The present data of interhemispheric asymmetry of the visual cortex supplement those of previous studies: areas BA17 and BA18 extend more medially and caudally in the left hemisphere than in the right (Amunts et al., 2000). Analyses of petalia also demonstrated interhemispheric differences in occipital protrusions (Zilles et al., 1996). In addition, interhemispheric, but not gender, differences have been observed in area 17 of the rat cortex (Galaburda et al., 1986).

The small but significant right-larger-than-left GLI values in $\mathrm{V} 5 / \mathrm{MT}+, \mathrm{BA} 17$, and BA18 indicate that the left hemisphere has more neuropil in all three visual areas than does the right hemisphere, regardless of gender. Our findings do not rule out the possibility that gender-related differences exist on a finer microstructural level, in individual layers, or with respect to glial cell number, for example (Galaburda et al., 1986; Reid and Juraska, 1992; Seymoure and Juraska, 1992), but they show that volumetric differences do not stem from gross differences in volume fractions of cell bodies across all cell layers. 
Interhemispheric differences in the volume fraction of cell bodies have been found previously in the presumed hand region of the primary motor cortex, BA4, in which a left-greater-thanright asymmetry of neuropil was observed but in which genderrelated differences were not present (Amunts et al., 1996). That study also analyzed the depths of the central sulcus in the motor hand region in a sample of in vivo MR datasets of tested righthanded subjects and demonstrated that the central sulcus was deeper on the left than right side. Both findings, postmortem and in vivo, have been interpreted as showing more space for connections in the hemisphere of the dominant hand. The similar direction of interhemispheric asymmetry in the amount of neuropil in BA4 of the motor cortex and V5/MT,$+ \mathrm{BA} 17$, and BA18 of the visual cortex is consistent with a relationship between handedness and eye dominance (Bourassa et al., 1996; McManus et al., 1999) or may represent a broader hemispheric dissimilarity.

Combining the gender equivalent GLI values with asymmetric volumetric data suggests that a higher proportion of the right female V5/MT + is involved with processing direct visual information from BA17 and BA18 than is that of males. At the same time, the larger absolute volume of the male V5/MT+ may indicate that males may process the same (or more) absolute amount of visual information. Another way to state this is that the male $\mathrm{V} 5 / \mathrm{MT}+$ has relatively and absolutely more space to process information from regions outside BA17/BA18, which may support the superior visuospatial processing observed in some cognitive tasks. Because males and females resemble each other in other motion tasks (Eals, 1986; Wendt and Risberg, 1994; Peters et al., 1995; Tran et al., 1998), we may assume the presence of additional neural strategies and their anatomical correlates.

\section{References}

Aboitiz F, Scheibel AB, Fisher RS, Zaidel E (1992) Fiber composition of the human corpus callosum. Brain Res 598:143-153.

Amunts K, Schlaug G, Schleicher A, Steinmetz H, Dabringhaus A, Roland PE, Zilles K (1996) Asymmetry in the human motor cortex and handedness. NeuroImage 4:216-222.

Amunts K, Malikovic A, Mohlberg H, Schormann T, Zilles K (2000) Brodmann's areas 17 and 18 brought into stereotaxic space: where and how variable? NeuroImage 11:66-84.

Amunts K, Kedo O, Kindler M, Pieperhoff P, Schneider F, Mohlberg H, Habel U, Shah JN, Zilles K (2005) Cytoarchitectonic mapping of the human amygdala, hippocampal region and entorhinal cortex. Anat Embryol 210:343-352.

Andreassi JL, Juszczak NM (1982) Hemispheric sex differences in response to moving stimuli as indicated by visual evoked potentials. Int J Neurosci 17:83-91.

Annese J, Gazzaniga MS, Toga AW (2005) Localization of the human cortical visual area MT based on computer aided histological analysis. Cereb Cortex 15:1044-1053.

Barequet G, Doodrich MT, Levi-Steiner A, Steiner D (2004) Contour interpolation by straight skeletons. Graph Models 66:245-260.

Barnikol UB, Amunts K, Dammers J, Mohlberg H, Fieseler T, Malikovic A, Zilles K, Niedeggen M, Tass P (2006) Pattern reversal visual evoked responses of V1/V2 and V5/MT as revealed by MEG combined with probabilistic cytoarchitectonic maps. NeuroImage 31:86-108.

Biasotti S (2004) Reeb graph representation of surfaces with boundary. Presented at the International Conference on Shape Modeling and Applications, Genoa, Italy, June.

Biasotti S (2005) Topological coding of surfaces with boundary using Reeb graphs. Comput Graph Geometry 7:31-45.

Bishop KM, Wahlsten DOUG (1997) Sex differences in the human corpus callosum: myth or reality? Neurosci Biobehav Rev 21:581-601.

Blinkov SM, Glezer II (1968) Das Zentralnervensystem in Zahlen und Tabellen. Jena, Germany: Fischer.

Boissonnat JD (1988) Shape reconstruction from planar cross sections. Comp Vison Graphics Image Process 44:1-29.
Born RT, Bradley DC (2005) Structure and function of visual area MT. Annu Rev Neurosci 28:157-189.

Bourassa DC, McManus IC, Bryden MP (1996) Handedness and eye dominance: a meta analysis of their relationship. Laterality 1:5-34.

Brodmann K (1909) Vergleichende Lokalisationslehre der Großhirnrinde in ihren Prinzipien dargestellt auf Grund des Zellenbaues. Leipzig, Germany: Barth.

Clarke S (1993) Callosal connections and functional subdivision of the human occipital lobe. In: Functional organization of the human visual cortex (Gulyas B, Ottoson D, Roland PE, eds), pp 137-149. Oxford: Pergammon.

Cohn NB, Kircher J, Emmerson RY, Dustman RE (1985) Pattern reversal evoked potentials: age, sex and hemispheric asymmetry. Electroencephalogr Clin Neurophysiol 62:399-405.

Davatzikos C, Resnick SM (1998) Sex differences in anatomic measures of interhemispheric connectivity: correlations with cognition in women but not in men. Cereb Cortex 8:635-640.

de Lacoste M-C, Horvath DS, Woodward DJ (1991) Possible sex differences in the developing human fetal brain. J Clin Exp Neuropsychol 13:831-846.

de Lacoste-Utamsing C, Holloway RL (1982) Sexual dimorphism in the human corpus callosum. Science 216:1431-1432.

Eals M (1986) Asymmetric processing in perception of apparent motion. Neuropsychologia 25:429-434.

Fahle M, Palm G (1983) Calculation of surface area from serial sections. J Neurosci Methods 9:75-85.

Fellemann DJ, van Essen DC (1991) Distributed hierarchical processing in the primate cerebral cortex. Cereb Cortex 1:1-47.

Fischl B, Sereno MI, Dale AM (1999) Cortical surface-based analysis. II. Inflation, flattening, and a surface-based coordinate system. NeuroImage 9:195-207.

Galaburda AM, Aboitiz F, Rosen GD, Sherman GF (1986) Histological asymmetry in the primary visual cortex of the rat: implications for mechanisms of cerebral asymmetry. Cortex 22:151-160.

Geiger B (1993) Three dimensional modeling of human organs and its application to diagnosis and surgical planning, pp 119. Paris: INRIA.

Good CD, Johnsrude I, Ashburner J, Henson RNA, Friston KJ, Frackowiak RSJ (2001) Cerebral asymmetry and the effects of sex and handedness on brain structure: a voxel-based morphometric analysis of 465 normal adult human brains. NeuroImage 14:685-700.

Grön G, Wunderlich AP, Spitzer M, Tomczak R, Riepe MW (2000) Brain activation during human navigation: gender-different neural networks as substrate of performance. Nat Neurosci 3:404-408.

Haug H (1980) Die Abhängigkeit der Einbettungsschrumpfung des Gehirngewebes vom Lebensalter. Verh Anat Ges 74:699-700.

Huk AC, Dougherty RF, Heeger DJ (2002) Retinotopy and functional subdivision of human areas MT and MST. J Neurosci 22:7195-7205.

Johnson BW, McKenzie KJ, Hamm JP (2002) Cerebral asymmetry for mental rotation: effects of response hand, handedness and gender. NeuroReport 13:1929-1932.

Kaufmann C, Elbel G-K, Gössl C, Pütz B, Auer DP (2001) Frequency dependence and gender effects in visual cortical regions involved in temporal frequency pattern processing. Hum Brain Mapp 14:28-38.

Kovalev VA, Kruggel F, von Cramon DY (2003) Gender and age effects in structural brain asymmetry as measured by MRI texture analysis. NeuroImage 19:895-905.

Lerch JP, Evans AC (2005) Cortical thickness analysis examined through power analysis and a population simulation. NeuroImage 24:163-173.

Lewis JW, van Essen DC (2000) Corticocortical connections of visual, sensorimotor, and multimodal processing areas in the parietal lobe of the macaque monkey. J Comp Neurol 428:112-137.

Luders E, Narr KL, Zaidel E, Thompson PM, Jancke L, Toga AW (2005) Parasagittal asymmetries of the corpus callosum. Cereb Cortex 16:1232-1238.

Macdonald D, Kabani N, Avis D, Evans AC (2000) Automated 3-D extraction of inner and outer surfaces of cerebral cortex from MRI. NeuroImage 12:340-356.

Malcolm CA, McCulloch DL, Shepherd AJ (2002) Pattern-reversal visual evoked potentials in infants: gender differences during early visual maturation. Dev Med Child Neurol 44:345-351.

Malikovic A, Amunts K, Schleicher A, Mohlberg H, Eickhoff SB, Wilms M, Palomero-Gallagher N, Armstrong E, Zilles K (2007) Cytoarchitectonic 
analysis of the human extrastriate cortex in the region of V5/MT+: a probabilistic, stereotaxic map of area hOc5. Cereb Cortex, in press.

Maunsell JH, van Essen DC (1983) The connections of the middle temporal visual area (MT) and their relationship to a cortical hierarchy in the macaque monkey. J Neurosci 3:2563-2586.

McManus IC, Porac C, Bryden MP, Boucher R (1999) Eye-dominance, writing hand, and throwing hand. Laterality 2:173-192.

Meyers D (1994) Reconstruction of surfaces from planar contours, pp 137. Washington, DC: University of Washington.

Meyers D, Skinner S, Sloan K (1991) Surfaces from contours: the correspondence and branching problems, Proceedings of Graphical Interface, pp 246-254. Toronto: Canadian Information Processing Society.

Muckli L, Kohler A, Kriegeskorte N, Singer W (2005) Primary visual cortex activity along the apparent-motion trace reflects illusory perception. PLoS Biol 3:e265.

Murphy DGM, DeCarli C, McIntosh AR, Daly E, Mentis MJ, Pietrini P, Szczepanik J, Schapiro MB, Grady CL, Horwitz B, Rapoport SI (1996) Sex differences in human brain morphometry and metabolism: an in vivo quantitative magnetic resonance imaging and positron emission tomography study on the effect of aging. Arch Gen Psychiatry 53:585-594.

Newsome WT, Allman JM (1980) Interhemispheric connections of the visual cortex in the owl monkey, Aotus trivirgatus, and the bushbaby. J Comp Neurol 194:209-233.

Orban GA (1997) Visual processing in macaque area MT/V5 and its satellites (MSTd and MSTv). In: Extrastriate cortex in primates (Rockland KS, Kaas J, Peters A, eds), pp 359-434. New York: Plenum.

O'Rourke J (1998) Computational geometry in C. Cambridge, UK: Cambridge UP.

Pascual-Leone A, Walsh V (2001) Fast backprojections from the motion to the primary visual area necessary for visual awareness. Science 292:510-512.

Peters M, Chrisholm P, Laeng B (1995) Spatial ability, student gender, and academic performance. J Engin Educ 84:69-73.

Rahman Q, Andersson D, Govier E (2005) A specific sexual orientationrelated difference in navigation strategy. Behav Neurosci 119:311-316.

Reeb G (1946) Sur les points singuliers d'une forme de Pfaff complètement intégrable ou d'une fonction numérique, pp 847-849. Paris: Comptes Rendus de L'Académie Sciences.

Reid SNM, Juraska JM (1992) Sex differences in neuron number in the binocular area of the rat visual cortex. J Comp Neurol 321:448-455.

Rodman HR, Gross CG, Albright TD (1990) Afferent basis of visual response properties in area MT of the macaque. II. Effects of superior colliculus removal. J Neurosci 10:1154-1164.

Rottschy C, Eickhoff SB, Schleicher A, Mohlberg H, Zilles K, Amunts K (2007) The ventral visual cortex in humans: cytoarchitectonic mapping of two extrastriate areas. Hum Brain Mapp, in press.

Schenk T, Ellison A, Rice N, Milner AD (2005) The role of V5/MT+ in the control of catching movements: an rTMS study. Neuropsychologia 43:189-198.

Schleicher A, Zilles K (1990) A quantitative approach to cytoarchitectonics: analysis of structural inhomogeneities in nervous tissue using an image analyser. J Microsc 157:367-381.

Schleicher A, Amunts K, Geyer S, Morosan P, Zilles K (1999) Observerindependent method for microstructural parcellation of cerebral cortex: a quantitative approach to cytoarchitectonics. NeuroImage 9:165-177.

Seymoure P, Juraska JM (1992) Sex differences in cortical thickness and the dendeitic tree in the monocular and binoclular subfields of the rat visual cortex at weaning age. Dev Brain Res 69:185-189.

Shipp S (2001) Corticopulvinar connections of areas V5, V4, and V3 in the macaque monkey: a dual model of retinal and cortical topographies. J Comp Neurol 439:469-490.

Shipp S, Zeki S (1989) The organization of connections between areas V5 and V1 in macaque monkey visual cortex. Eur J Neurosci 1:309-332.
Sillito AM, Jones HE (2002) Corticothalamic interactions in the transfer of visual information. Philos Trans R Soc Lond B Biol Sci 357:1739-1752.

Silvanto J, Lavie N, Walsh V (2005) Double dissociation of V1 and V5/MT activity in visual awareness. Cereb Cortex 15:1736-1741.

Sincich LC, Park KF, Wohlgemuth MJ, Horton JC (2004) Bypassing V1: a direct geniculate input to area MT. Nat Neurosci 7:1123-1128.

Skullerud K (1985) Variations in the size of the human brain. Acta Neurol Scand 71:1-94.

Tootell RBH, Taylor JB (1995) Anatomical evidence for MT and additional cortical visual areas in humans. Cereb Cortex 5:39-55.

Tran DB, Silverman SE, Zimmerman K, Feldon SE (1998) Age-related deterioration of motion perception and detection. Graefes Arch Clin Exp Ophthalmol 236:269-273.

Ungerleider L, Mishkin M (1982) Two cortical visual systems. In: Analysis of visual behavior (Ingle DG, Goodale MA, Mansfield RJQ, eds), pp 549586. Cambridge, MA: MIT.

Ungerleider LG, Haxby JV (1994) "What" and "where" in the human brain. Curr Opin Neurobiol 4:157-165.

Uylings HBM, von Eden CG, Hofman MA (1986) Morphometry of size/ volume variables and comparison of their bivariate relations in the nervous system under different conditions. J Neurosci Methods 18:19-37.

van Essen DC, Maunsell JH, Bixby JL (1981) The middle temporal visual area in the macaque: myeloarchitecture, connections, functional properties and topographic organization. J Comp Neurol 199:293-326.

van Essen DC, Newsome WT, Maunsell JHR, Bixby JL (1986) The projections from striate cortex (V1) to areas V2 and V3 in the macaque monkey: asymmetries, areal boundaries, and patchy connections. J Comp Neurol 244:451-480

van Essen DC, Drury HA, Joshi S, Miller MI (1998) Functional and structural mapping of human cerebral cortex: solutions are in the surfaces. Proc Natl Acad Sci USA 95:788-795.

van Essen DC, Drury HA, Dickson J, Harwell J, Hanlon D, Anderson CH (2001) An integrated software suite for surface-based analyses of cerebral cortex. J Am Med Inform Assoc 8:443-459.

Vierordt H (1893) Anatomische, physiologische und physikalische Daten und Tabellen zum Gebrauch für Mediziner. Jena, Germany: Fischer Verlag.

von Economo C, Koskinas GN (1925) Die Cytoarchitektonik der Hirnrinde des erwachsenen Menschen. Berlin: Springer.

Walters NB, Eickhoff SB, Schleicher A, Zilles K, Amunts K, Egan GF, Watson JDG (2007) Observer-independent analysis of high-resolution MR images of the human cerebral cortex: in vivo delineation of cortical areas. Hum Brain Mapp 28:1-8.

Wendt PE, Risberg J (1994) Cortical activation during visual spatial processing: Relation between hemispheric asymmetry of blood flow and performance. Brain Cogn 24:87-103.

Wilms M, Eickhoff SB, Specht K, Amunts K, Malikovic A, Zilles K, Fink GR (2005) Human V5/MT+: comparison of functional and cytoarchitectonic data. Anat Embryol 210:485-495.

Yoshida K, Benevento LA (1981) The projection from the dorsal lateral geniculate nucleus of the thalamus to extrastriate visual association cortex in the macaque monkey. Neurosci Lett 22:103-108.

Zeki S, Watson JD, Lueck CJ, Friston KJ, Kennard C, Frackowiak RS (1991) A direct demonstration of functional specialization in human visual cortex. J Neurosci 11:641-649.

Zilles K, Dabringhaus A, Geyer S, Amunts K, Qü M, Schleicher A, Gilissen E, Schlaug G, Seitz R, Steinmetz H (1996) Structural asymmetries in the human forebrain and the forebrain of non-human primates and rats. Neurosci Behav Rev 20:593-605.

Zilles K, Schleicher A, Palomero-Gallagher N, Amunts K (2002) Quantitative analysis of cyto- and receptor architecture of the human brain. In: Brain mapping: the methods (Mazziotta JC, Toga A, eds), pp 573-602. Amsterdam: Elsevier. 